\title{
Biomechanical Comparison Between Bicortical Pin and Monocortical Screw/Polymethylmethacrylate Constructs in the Cadaveric Canine Cervical Vertebral Column
}

\author{
Bianca F. Hettlich Dr. med vet, Diplomate ACVs ${ }^{1,}{ }^{*}$, Matthew J. Allen Vet MB, PhD ${ }^{1}$, \\ Daniel Pascetta BS ${ }^{1}$, Geoffrey T. Fosgate DVM, PhD, Diplomate ACVPM ${ }^{2}$ and
}

Alan S. Litsky MD, Sc.D. ${ }^{3,4}$

\begin{abstract}
${ }^{1}$ Department of Veterinary Clinical Sciences, College of Veterinary Medicine, Ohio State University, Columbus, $\mathrm{OH}$

${ }^{2}$ Department of Production Animal Studies, Faculty of Veterinary Science, University of Pretoria, South Africa

${ }^{3}$ Department of Orthopaedics, College of Medicine, Ohio State University, Columbus, $\mathrm{OH}$

${ }^{4}$ Department of Biomedical Engineering, College of Engineering, Ohio State University, Columbus, $\mathrm{OH}$
\end{abstract}

\begin{abstract}
Objective

To compare biomechanical stiffness of cadaveric canine cervical spine constructs stabilized with bicortical stainless steel pins and polymethylmethacrylate (PMMA), monocortical stainless steel screws with PMMA, or monocortical titanium screws with PMMA.
\end{abstract}

\section{Study Design}

Biomechanical cadaver study.

\section{Animals}

Eighteen canine cervical vertebral columns (C2-C7) were collected from skeletally mature dogs (weighing 22-32 kg).

\section{Methods}

Specimens were radiographed and examined by dual energy X-ray absorptiometry. Stiffness of the unaltered $\mathrm{C4}-\mathrm{C} 5$ intervertebral motion unit was measured in extension, flexion and lateral bending using non-destructive 4-point bend testing. Specimens were then stabilized by (1) bicortical stainless steel pins/PMMA, (2) monocortical stainless steel screws/PMMA, or (3) monocortical titanium screws/PMMA. Mechanical testing was repeated and stiffness data from unaltered specimens and the 3 treatment groups were compared. 


\section{Results}

All 3 surgical methods significantly increased stiffness of the C4-C5 motion unit compared with the unaltered specimen ( $P<.001$ for all treatments), but stiffness was not significantly different among the 3 fixation groups $(P=.578)$.

\section{Conclusions}

In this model, monocortical screw fixation (with stainless steel or titanium screws) was biomechanically equivalent to bicortical fixation.

Canine cervical vertebral column stabilization is commonly used to treat instability caused by trauma and cervical spondylomyelopathy (CSM).[1-4] Placement of bicortical pins into adjacent vertebrae and fixation with polymethylmethacrylate (PMMA) is an established surgical technique for cervical vertebral column stabilization.[5] This construct has been tested biomechanically and eliminates motion at the stabilized intervertebral motion unit.[6] Unfortunately, it is associated with a great potential for iatrogenic trauma to neurovascular structures. Bicortical pin insertion even at the recommended angles of 30$40^{\circ}$ carries an unacceptably high risk of canal violation. [7] Because of the narrow safe implant corridor, injury to vertebral canal and spinal cord, intervertebral foramen and nerve roots, or vertebral foramen and vertebral artery are likely. Ventral bicortical screw fixation using the cervical transverse processes and fixation with metal bar reinforced PMMA compared favorably to published data for bicortical pin/PMMA fixation. [8] Use of a locking compression plate with monocortical screw fixation has been evaluated biomechanically but without direct comparison to other cervical stabilization techniques. [9] Reports of clinical use of monocortical screw constructs in dogs with CSM suggest that monocortical screw fixation is effective in stabilizing the cervical spine[1, 4, 10-13]; however, none of these constructs have been evaluated biomechanically and compared to the established bicortical pin fixation.

The gold standard for spinal cord imaging in veterinary medicine has evolved from myelography and computed tomography (CT) to magnetic resonance imaging (MRI) as MRI has become increasingly available. Metal artifacting occurs during MRI with the use of ferromagnetic and non-ferromagnetic implants; however, artifact with non-ferromagnetic metals such as titanium can be minimized with certain MRI parameters. [14-16] Both titanium alloy and pure titanium implants decrease the amount of metal artifacts during MRI compared to stainless steel in human spine studies and improve evaluator assessment of neurovascular structures. $[17,18]$ This would make titanium the preferred implant for veterinary use when postoperative MRI is desirable. Mechanical properties of titanium and stainless steel pedicle screw constructs for human vertebral column stabilization have been compared.[19, 20] Biomechanical studies evaluating the use of titanium screws for vertebral column stabilization in dogs are lacking.

Our purpose was to compare biomechanical properties of the traditional bicortical stainless steel pin/PMMA construct to 2 unicortical screw/PMMA constructs, 1 using stainless steel screws, the other titanium alloy screws in cadaveric canine cervical vertebral columns. Our 
hypothesis was that there would be no significant difference in the stiffness among the 3 surgical constructs.

\section{MATERIALS AND METHODS}

\section{Vertebral Specimens}

The study was approved by The Ohio State University Institutional Laboratory Animal Care and Use Committee. Canine cervical vertebral columns (C2-C7) of mature dogs ( $n=18)$ euthanatized for reasons unrelated to this study were collected. To be included, dogs had to weigh 22-32 kg and be mature based on dentition. Lateral and dorsoventral radiographic projections were obtained to ensure physeal closure and lack of vertebral column deformities or other pre-existing conditions affecting the vertebrae and disk spaces. Vertebral columns from immature dogs were excluded as were specimens with apparent bony abnormalities on radiographs. Cervical vertebral columns had dual-energy X-ray absorptiometry (DEXA) scans (Lunar Prodigy; GE Healthcare, Milwaukee, WI) to determine bone mineral density of $\mathrm{C} 4$ and $\mathrm{C} 5$ vertebral bodies. Specimens were sorted into balanced groups based on these mineral density values. To prepare specimens for testing, surrounding soft tissues were resected except for vertebral musculature, joint capsules and ligaments associated with vertebrae C3-C6. Specimens were then wrapped in moist towels soaked in sterile saline $(0.9 \% \mathrm{NaCl})$ solution and frozen at $-20^{\circ} \mathrm{C}$ until testing. Specimens were kept moist using sterile saline solution during processing and testing.

\section{Biomechanical Data Collection}

The vertebral motion units (VMUs) between C3-C4 and C5-C6 were stabilized using transarticular cross-pins that did not interfere with motion at C4-C5. C2, C7, and the cranial and caudal aspect of C3 and C6, respectively, were freed of soft tissues and augmented with wood screws to improve stability within the potting cement (Bondo, 3M; Lowes, Columbus, $\mathrm{OH}$ ) applied to the cranial and caudal end of the vertebral section. Specimens were potted leaving the caudal half of $\mathrm{C} 3$ to the cranial half of $\mathrm{C} 6$ and surrounding soft tissues exposed. $\mathrm{K}$-wires were placed on midline in the dorsal and lateral plane in $\mathrm{C} 4$ and $\mathrm{C} 5$ to affix the extensometer.

Each specimen was allowed to thaw to room temperature and tested in extension, flexion and right lateral bending using a custom made four-point bending fixture (1). After being placed in the jig in a neutral position, a preload of 5 Newtons $(\mathrm{N})$ was applied to stabilize the specimen and assure that all specimen tests were initiated under the same condition. Testing was load-controlled at $50 \mathrm{~N} / \mathrm{min}$ to $150 \mathrm{~N}$ in flexion and extension and to $100 \mathrm{~N}$ in right lateral bending based on pilot data. Each specimen sequentially underwent 4 full cycles of extension, flexion, and lateral bending. Between each cycle, specimens were allowed to rest in neutral for 30 seconds to allow for tissue recovery. Actuator displacement $(\mathrm{mm})$, applied load $(\mathrm{N})$, and extensometer displacement $(\mathrm{mm})$ data were collected continually and recorded at $0.5 \mathrm{~N}$ intervals using the data acquisition system integral with the servo hydraulic test frame (MTS Bionix 858 Test System, MTS, Eden Prairie, MN). Load and extensometer displacement data from the 4th cycle of each loading direction were used to calculate load-displacement curves for each bending moment of the unaltered and 
instrumented C4-C5 motion unit. Stiffness $(\mathrm{Nm} / \mathrm{m})$ was calculated by selecting the linear portion of each load-displacement curve. The same loading and data collection protocol was used for unaltered and surgically fixated vertebral columns.
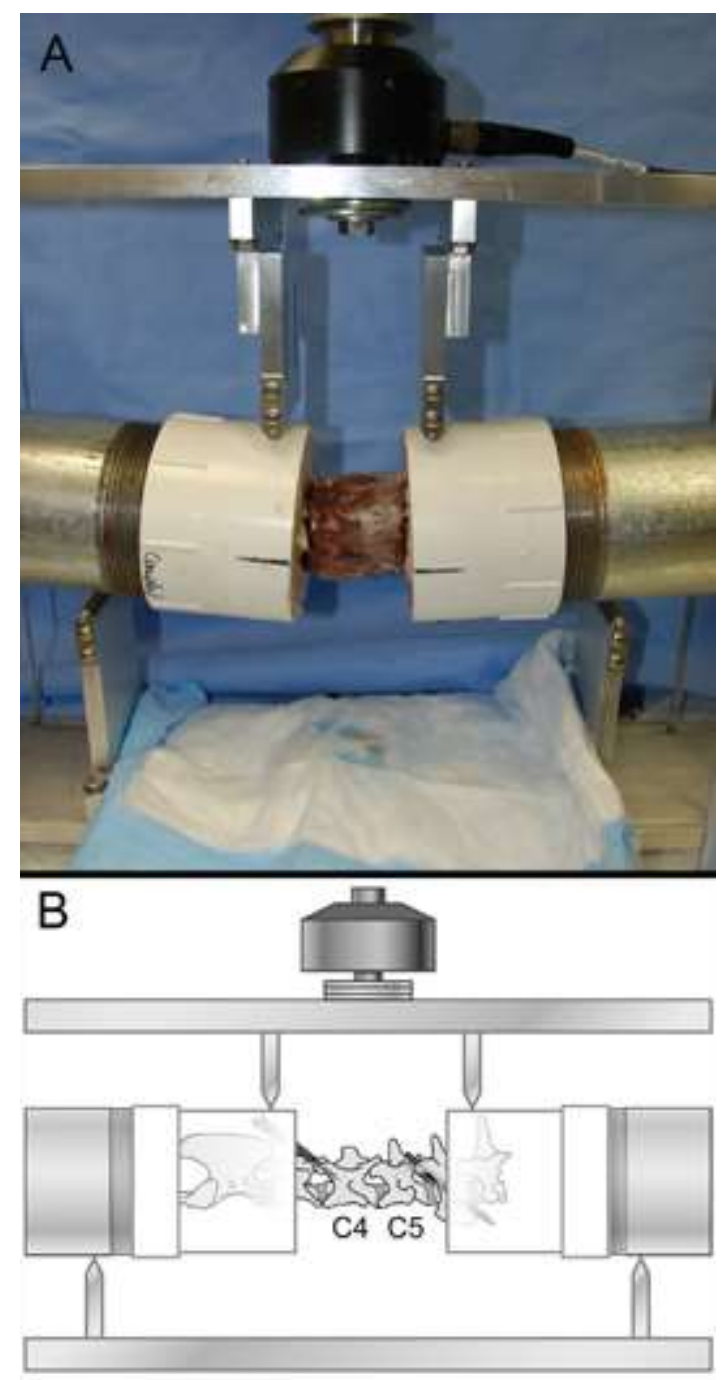

Figure 1. Photograph (A) and illustration (B) of the 4-point bending fixture used to evaluate stiffness of intact and surgically fixed canine cadaveric cervical vertebral columns.

\section{Surgical Fixation}

After mechanical testing of the unaltered specimen, the longus colli musculature was resected in an $\sim 5 \mathrm{~cm}$ long by $3.5 \mathrm{~cm}$ wide area centered over the C4-C5 intervertebral disk, which remained unaltered. The area of resected musculature was kept as uniform as possible between specimens and served as a mold for the PMMA. Specimens were instrumented according to 3 fixation groups (2). 


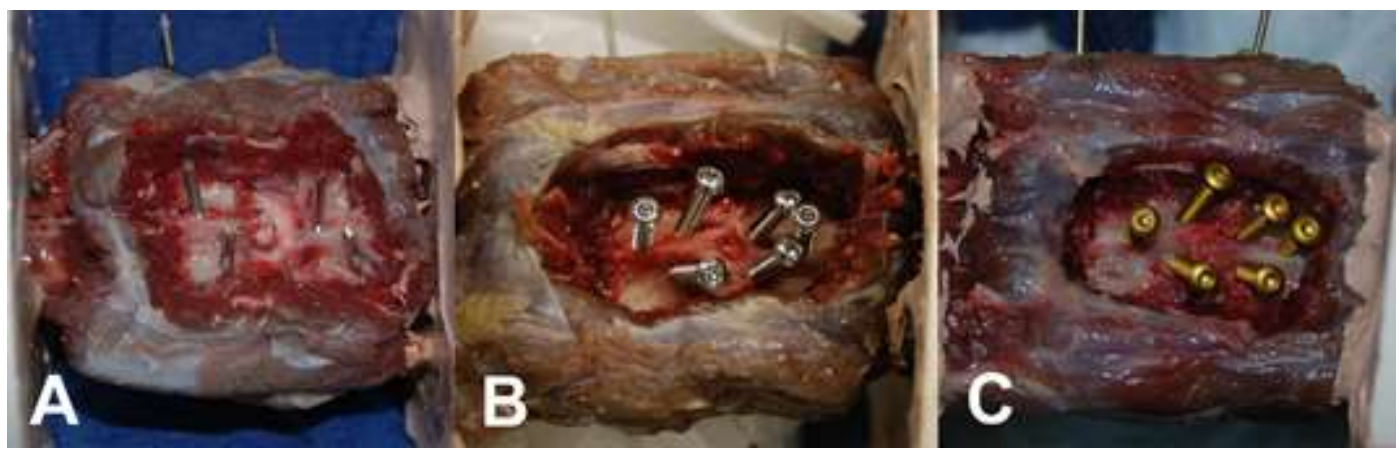

Figure 2. Photographs of the ventral aspect of cadaveric cervical vertebral specimens according to fixation groups before polymethylmethacrylate application: (A) bicortical stainless steel pins, (B) monocortical stainless steel screws, and $(C)$ monocortical titanium screws.

\section{Group 1-Bicortical Stainless Steel Positive-Profile Pins With PMMA}

Two $1 / 8$ inch positive profile stainless steel cortical pins were placed into each vertebral body of $\mathrm{C} 4$ and $\mathrm{C5}$.[6] Pin placement was started on ventral midline and angled $30-40^{\circ}$ from the sagittal plane into the vertebral pedicle with the goal of engaging the transcortex.[21, 22] Pins were cut with $12-15 \mathrm{~mm}$ protruding from the ventral vertebral body surface to allow incorporation into bone cement.

\section{Group 2-Monocortical Stainless Steel Cortical Screws With PMMA}

Six $3.5 \mathrm{~mm}$ stainless steel cortical screws (Synthes Vet, West Chester, PA) were inserted into the mid-body (1) and caudal metaphyseal region (2) of C4 and cranial metaphyseal region (2) and mid-body (1) of C5. For each screw, a hole was drilled into the ciscortex with a $2.5 \mathrm{~mm}$ drill bit and tapped with a $3.5 \mathrm{~mm}$ thread tap. Orientation of the screws was caudoventral to craniodorsal with the goal to be parallel to the vertebral endplate orientation for all but the most caudal screw. The latter one was oriented in a cranioventral to caudodorsal direction toward the caudal endplate of $\mathrm{C} 5$ because of physical obstruction by the specimen potting construct. The goal was to position screws in monocortical fashion to a depth where they contacted the inner cortex of the vertebral canal. Screws protruded 12-15 $\mathrm{mm}$ from the ventral vertebral body surface to allow incorporation into the bone cement.

\section{Group 3-Monocortical Self-Tapping Titanium Alloy Cortical Screws With PMMA}

Six $3.5 \mathrm{~mm}$ self-tapping titanium alloy cortical screws (Synthes) were applied similarly to group 2, except that holes were not tapped.

Implant placement was performed by the same individual for all specimens. During pin placement, a goniometer was used to apply bicortical pins within the recommended range of angles (goal of $35^{\circ}$ ). When drilling for monocortical screws, the drill was stopped when the bit was felt to penetrate the ciscortex of the vertebral body. No special drill guide or drill stop was used to limit inadvertent penetration through the transcortex with the drill bit. Regardless of fixation group, $20 \mathrm{~g}$ PMMA (Simplex P Bone Cement, Stryker, Mahwah, NJ) were mixed according to manufacturer's specification and applied around implants to create 
a uniform cement mantle. Cement was allowed to harden for a minimum of 20 minutes before retesting.

\section{Postoperative Implant Assessment}

Post-testing, orthogonal radiographic projections were used to assess implant position and any bony damage from mechanical testing (3). Vertebral columns were then cleared of remaining soft tissues using a dermestid beetle colony. Implant position in relation to the vertebral canal and intervertebral and vertebral foramina was assessed. Pin violation was characterized as partial or complete if part or the entire circumference of the pin penetrated the canal or foramina, respectively. In the monocortical screw fixation groups, vertebrae were evaluated for implant violation of the vertebral canal and further characterized as cortical lift (minor disturbance of cortical bone without visible implant), $\leq 1$, $\leq 2$, or $\geq 2 \mathrm{~mm}$ of screw protrusion. Five evaluators blinded to actual position of monocortical screws assessed implant position radiographically in regards to the vertebral canal. Results were evaluated based on consensus, which required $\geq 4 / 5$ evaluators to choose the same position (in or out). Screws causing cortical lift only were counted as both "in" and "out" in independent analyses concerning these data.

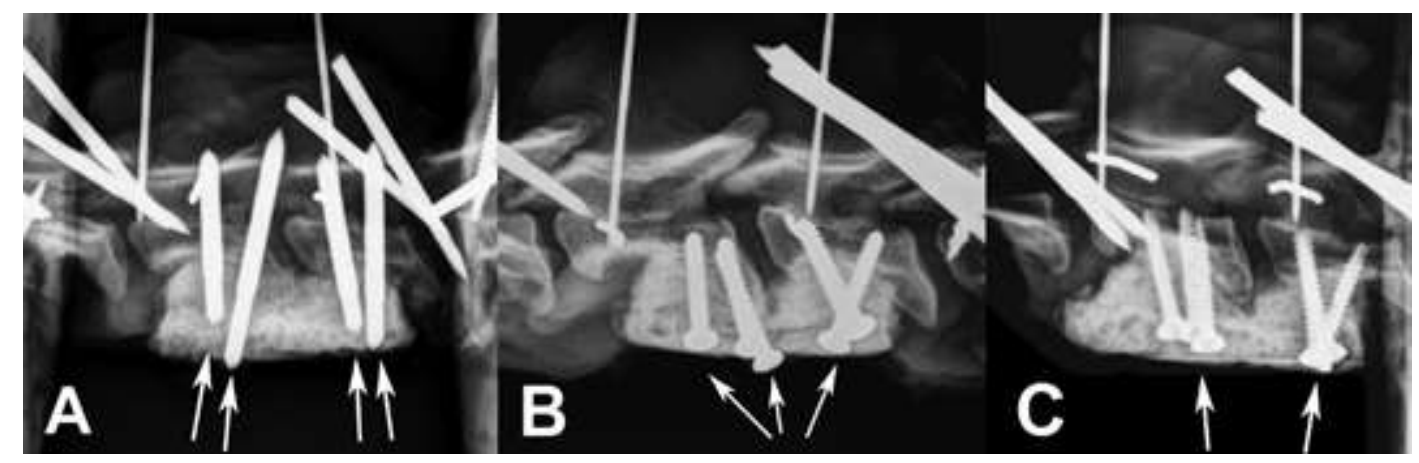

Figure 3. Lateral cervical vertebral column radiographs of fixation groups: (A) bicortical stainless steel pins, (B) monocortical stainless steel screws, and (C) monocortical titanium screws; arrows indicate construct implants-other pins and k-wires are part of the potting fixture or extensometer.

\section{Statistical Analysis}

Descriptive statistics were calculated for all variables including mean $( \pm S D)$, median, and range. Chi-square and ANOVA tests were used to identify differences among treatment groups for categorical and continuous data, respectively. Stiffness values were compared between unaltered and surgically stabilized specimens using a repeated measures ANOVA procedure with Bonferroni adjustment for post-hoc comparisons. The ANOVA model included factors related to the 3 treatment groups (bicortical pin/PMMA, monocortical stainless steel screw/PMMA, and monocortical titanium screw/PMMA) and the 3 positional measurements (i.e., extension, flexion, and lateral bending). A post-hoc power analysis was performed to determine the adequacy of the sample size to detect group differences in stiffness. Sensitivity for radiographic detection of a screw violating the vertebral canal was estimated as the proportion of screws truly violating identified by evaluator consensus. Specificity was similarly estimated as the proportion of screws not in the canal correctly identified by the consensus evaluation. Ninety-five percent mid-P exact confidence intervals 
(CI) were calculated for sensitivity and specificity estimates. Statistical testing was performed using commercially available software (IBM SPSS Statistics, Version 20, International Business Machines Corp., Armonk, NY) and significance was set at $P<.05$.

\section{RESULTS}

\section{Vertebral Specimens}

One specimen in the titanium screw/PMMA group was replaced after data collection was completed because of faulty extensometer recording during the last set of tests. All data for this vertebral column were excluded. The following represents results of the 18 included specimens. Dogs (13 intact and 2 neutered males, 3 intact females) ranged in weight from 22 to $32 \mathrm{~kg}$ (median, $26.5 \mathrm{~kg}$ ) and had no known history of vertebral column disease. Sixteen dogs were Pit Bulls or Pit Bull mix-breed dogs. All dogs were mature based on radiographic assessment and were free of obvious bony abnormalities involving the cervical vertebral column. Body weight, gender, and breed were not significantly different among treatment groups ( $P=.714, P=.301$, and $P=.570$, respectively). Bone mineral density for vertebral bodies $C 4$ and $C 5$ ranged from 0.899 to $1.346 \mathrm{~g} / \mathrm{cm}^{2}$ and $0.842-1.259 \mathrm{~g} / \mathrm{cm}^{2}$, respectively. The average for both vertebral bodies ranged from 0.853 to $1.3025 \mathrm{~g} / \mathrm{cm}^{2}$ (mean, $1.115 \mathrm{~g} / \mathrm{cm}^{2}$; median, $1.142 \mathrm{~g} / \mathrm{cm}^{2}$ ). Bone mineral density was not significantly different between treatment groups (median, $1.164 \mathrm{~g} / \mathrm{cm}^{2}$ for cross pins; $1.098 \mathrm{~g} / \mathrm{cm}^{2}$ for stainless steel screws; $1.141 \mathrm{~g} / \mathrm{cm}^{2}$ for titanium screws; $\left.P=.963\right)$. Bone density also did not vary by gender $(P=.863)$.

\section{Biomechanical Testing}

Mean $( \pm S D)$ difference in stiffness from pre- to post-fixation in extension was 304 (136) N/mm, $256(58) \mathrm{N} / \mathrm{mm}$ and 263 (67) N/mm for the bicortical stainless steel positiveprofile pins with PMMA (CP), monocortical stainless steel cortical screws with PMMA (SS), and monocortical self-tapping titanium cortical screws with PMMA (Ti) fixation groups, respectively. Mean (SD) difference in stiffness from pre- to post-fixation in flexion was 207 (67) N/mm, 220 (42) N/mm and 180 (53) N/mm for the CP, SS, and Ti fixation groups, respectively. Mean (SD) difference in stiffness from pre- to post-fixation in lateral bending was $224(75) \mathrm{N} / \mathrm{mm}, 261(86) \mathrm{N} / \mathrm{mm}$ ) and 185 (47) N/mm for the CP, SS, and Ti fixation groups, respectively. All surgical methods increased stiffness over the unaltered spine $(P<.001 ; 4)$. Stiffness did not vary by surgical method $(P=.578)$ but was different by directional measurement $(P=.001 ; 5)$. Stiffness was highest in extension and was significantly different than flexion values $(P=.002)$ but not lateral bending $(P=.101)$, nor was there a significant difference between flexion and lateral bending $(P=.533)$. Post-hoc power analysis determined that the sample size was adequate to detect a stiffness difference of $43.5 \mathrm{~N} / \mathrm{mm}$ between groups with $80 \%$ power. 


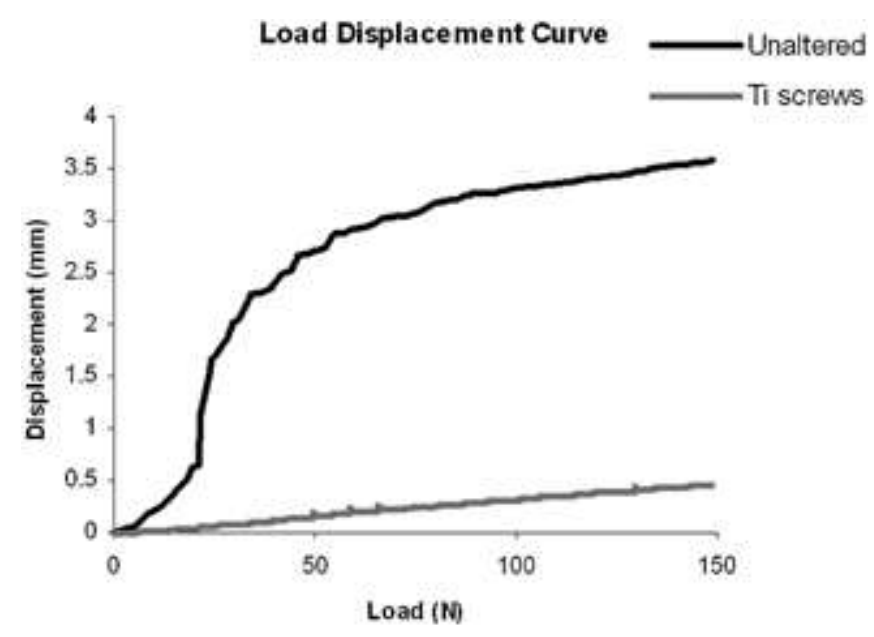

Figure 4. Representative load-displacement curve of a cervical vertebral specimen in its unaltered form (black) and after monocortical Titanium screw fixation (gray).

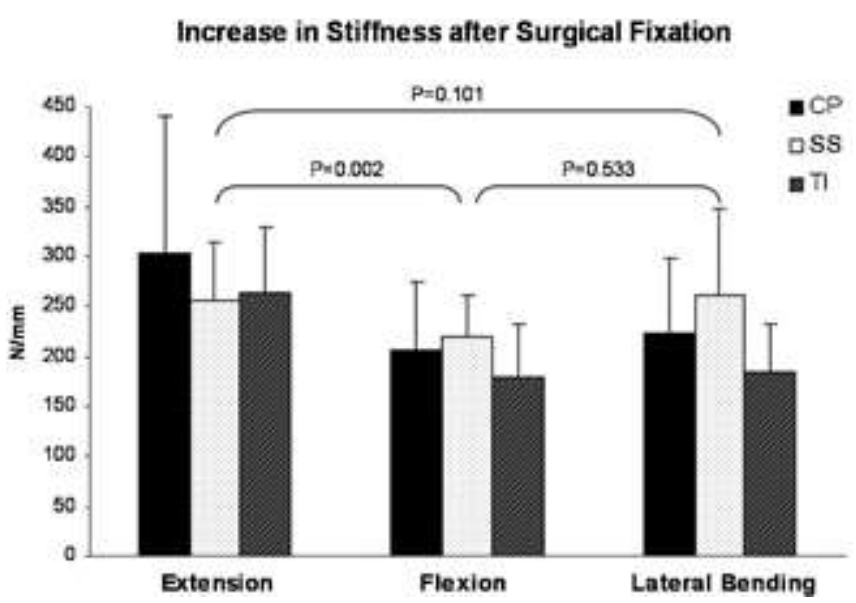

Figure 5. Graph showing the increase in stiffness compared to the unaltered vertebral columns for each surgical treatment group in each direction.

\section{Postoperative Implant Assessment}

None of the specimens had radiographically apparent failure of the implants or bone after biomechanical testing. Visual inspection of $\mathrm{C} 4$ and $\mathrm{C} 5$ after removal of soft tissues revealed that all 24 Steinman pins violated the cervical vertebral canal (4 complete, 20 partial; 6). Further, 5 of 6 specimens in the pin/PMMA group had 1 pin violate the intervertebral foramen ( 1 complete, 4 partial). Six of 12 spines with monocortical screw/PMMA constructs had some form of vertebral canal violation by one or more screw. Seven screws caused minor cortical lift and 7 screws penetrated into the canal (7). Of 36 monocortical stainless steel screws, 3 caused cortical lift and 2 penetrated $\leq 2 \mathrm{~mm}$ into the vertebral canal. Of the 36 monocortical titanium screws, 4 caused cortical lift, 3 penetrated $\leq 1 \mathrm{~mm}$, and 2 penetrated $\leq 2 \mathrm{~mm}$ into the vertebral canal. Of the 9 screws in the titanium group causing cortical lift or penetrating, 6 occurred in a single specimen. The most common location for inadvertent bicortical screw placement was the most cranial screw within C4, positioned in the middle of the vertebral body (4 of 7 penetrating screws). 

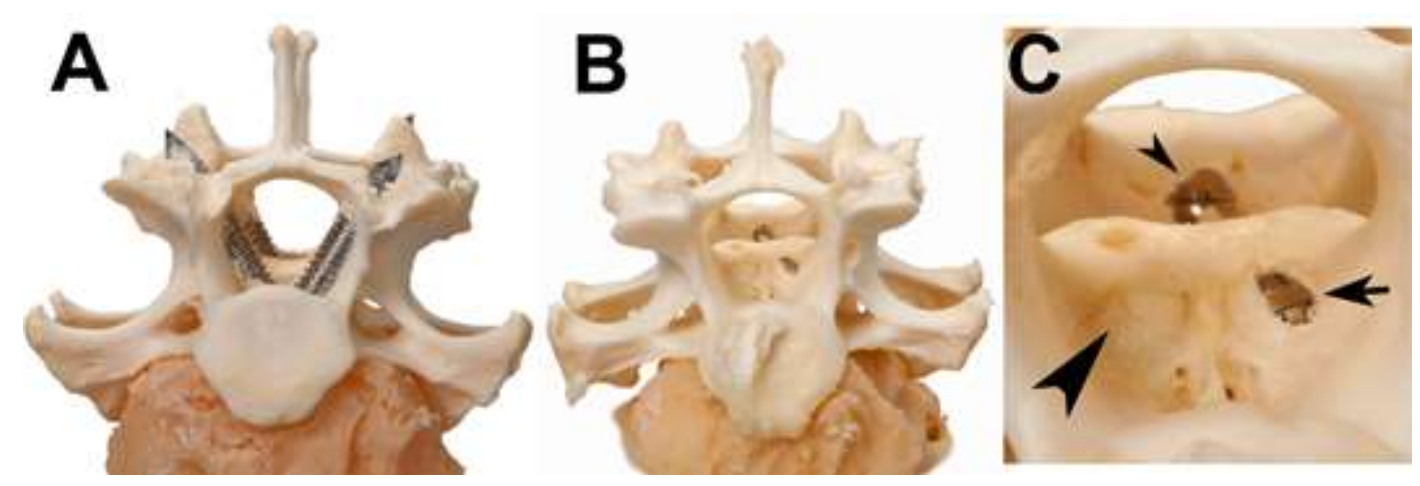

Figure 6. Photographs of vertebrae showing bicortical pin violation $(A)$ and minor protrusion of monocortical screws (B) with a close-up of protruding screws (C). The large black arrow head points to cortical lift caused by a screw. The black arrow and small arrow head point to screws protruding $<1$ and $<2 \mathrm{~mm}$, respectively.

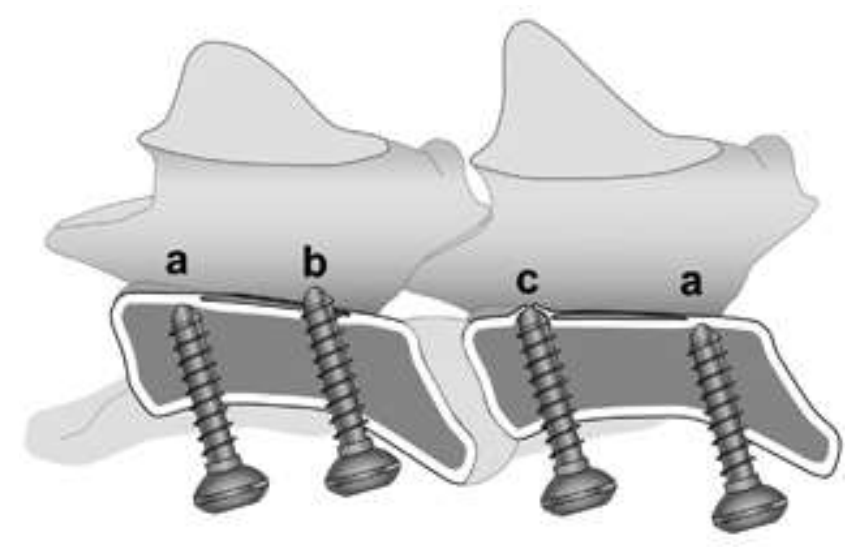

Figure 7. Line drawing of 2 cervical vertebrae showing screw placement in the vertebral body: (a) monocortical screws with screw tip touching transcortex, (b) screw protruding through transcortex, and (c) screw causing cortical lift of transcortex.

Consensus among evaluators regarding monocortical screw position on radiographs was high for screws that were either "in" or "out" with 63/65 (97\%) of screws correctly identified as either in or out. When screws causing cortical lift were included as "in" screws, consensus dropped to $64 / 72$ (89\%) of screws. Sensitivity for detecting screws violating the canal was estimated as 57\% (95\% Cl: 31-80\%) and $86 \%$ (95\% Cl: 47-99\%) with and without inclusion of screws causing cortical lift, respectively. Specificity was estimated as $97 \%$ (95\% Cl: 90-99\%) and $100 \%$ (95\% Cl: $95-100 \%)$ with and without inclusion of screws causing cortical lift, respectively.

\section{DISCUSSION}

We found that monocortical screw/PMMA constructs with either stainless steel or titanium alloy screws were biomechanically comparable to bicortical pin/PMMA constructs in the cadaveric canine cervical vertebral column. We also documented that $100 \%$ of bicortical pins violated the vertebral canal compared with a much lower incidence of violation in the screw fixation groups. 
Because of the anatomy of the canine cervical vertebral column, surgical stabilization poses several challenges. The bulk of bone lies within the vertebral body, which can be accessed with relative ease from a ventral approach; however, even bone purchase within the body is limited depending on whether implants engage bone close to the vertebral endplates (most bone) or toward the middle (narrow waist of bone). Landmarks and insertion angles for bicortical pin placement in the cervical spine have been reported.[5, 6] Biomechanical studies using bicortical pins with polymethylmethacrylate have shown a significant increase in stiffness of the instrumented intervertebral motion unit. [6, 8, 23] Unfortunately, the pedicle of the canine cervical vertebrae is narrow and in close proximity to the vertebral foramen, housing the vertebral artery. Corlazzoli found that regardless of the insertion angle, there is no "safe" implant corridor for bicortical pin placement in the canine cervical vertebral column as implants will either violate the vertebral canal or vertebral foramen.[7] Use of monocortical screws with plate fixation of the canine cervical vertebral column has been reported in clinical case series including plastic plates, bone plates, ComPact UniLock plates, spinal locking plates, and locking plates.[1, 4, 10-12] The proposed benefit of locking plates is the rigid fixation of the screw to the plate, thereby limiting motion of the screw within bone and implant leading to improved implant stability. $[9,10,24]$ However, screws can even loosen in implants designed to rigidly lock screws with the plate. In a prospective case series using cervical spine locking plates, 4/10 dogs had screw loosening and 2/10 plate shifting.[1] In a clinical report on the use of the ComPact UniLock plate system, 2/12 of dogs had screw loosening with implant failure, with 1 dog requiring revision using pins/PMMA. [4] Recently, locking compression plate (LCP) monocortical fixation has been biomechanically evaluated but was not compared to other fixation constructs such as pins/PMMA. [9] Using locking plates not designed for the use in the canine cervical vertebral column poses several limitations. Plate length and hole location may not be appropriate for a particular dog. The locking mechanism usually requires a specific screw orientation within the screw hole (typically perpendicular), limiting versatility to place screws in different orientations from the plate. Currently available cervical spine locking plates are produced for people and are often cost-prohibitive for dog-owners.[1, 10] Development of veterinary vertebral locking plates may offer the advantage of an implant designed for the canine cervical spine along with competitive pricing.

The monocortical screw/PMMA construct rigidly connects screw heads and part of the threads with cement, thereby acting similarly to a locking plate mechanism. Failure of such a construct is expected at the screw/bone interface or via fracturing of the PMMA. Our biomechanical data supports that monocortical screw/PMMA constructs behave similarly to bicortical pin/constructs. It is likely that, if all currently described monocortical fixation techniques were compared, no clinically important difference in stiffness would be observed in an in vitro model. Apart from the decreased risk of injury to neurovascular structures, the proposed benefits of a monocortical screw/PMMA construct include ease of placement of individual screws with a high degree of freedom regarding insertion location and angle, use of readily available implant components, and decreased cost for the owner compared to currently available cervical spine locking plates. Side effects from the use of PMMA have been described.[25-29] In a spinal fixation construct as described here, complications could arise from harmful effects during exothermic polymerization of the PMMA. Also, whereas PMMA in the viscous working phase provides great versatility during application, the ultimate thickness and bulk of the cement may affect adjacent soft tissues such as trachea 
and esophagus. The PMMA dimensions we used were relatively uniform with cement height of $1.5-2.0 \mathrm{~cm}$ as measured on cleaned specimens. In our constructs, $20 \mathrm{~g}$ PMMA covered screw heads and produced a subjectively appropriately sized amount of cement flush with the level of the longus colli musculature, thereby unlikely to interfere with adjacent structures.

In general, titanium alloy implants appear to have several advantages over stainless steel such as higher tensile and yield strength, lower modulus of elasticity, and superior strength under high cyclic loading.[30, 31] Spinal constructs implanted in people using titanium and stainless steel screws have been compared biomechanically and support the use of titanium alloy implants.[19, 20] However, whereas titanium alloy screws performed similarly to stainless steel screws in our study, their clinically more relevant advantage is likely the superior $\mathrm{MRI}$ and $\mathrm{CT}$ resolution with decreased interference compared to stainless steel implants. $[32,33]$ Our results support use of titanium screws in a monocortical construct, which should increase the versatility of postoperative imaging and improve spinal cord assessment because of improved MRI compatibility.

Our experimental approach differs from other reports. $[6,8,9,34]$ Pilot data were obtained using a previously published experimental set-up in which 3 vertebral motion units (VMU) $\mathrm{C} 3 / 4, \mathrm{C} 4 / 5$, and $\mathrm{C} 5 / 6$ were unconstrained within the potting fixture with $\mathrm{C} 4 / 5$ receiving instrumentation. [8] Despite trying to replicate this set-up we were unable to document a difference between unaltered and fixed spines. It was suspected that, within the chosen actuator displacement limit, the adjacent VMUs C3/4 and C5/6 were compensating for the instrumented segment of $C 4 / 5$. The experimental set-up was subsequently modified so that only $1 \mathrm{VMU}$ was unconstrained within the potting fixture (C4/5). Extensometers were also used to measure vertebral displacement directly rather than actuator displacement which could be falsely elevated by movement of the potting fixture. Pilot data using the new setup provided expected differences in stiffness and information concerning maximum safe loads for study specimens in extension, flexion and lateral bending. It is recognized that bone mineral density influences screw fixation strength, therefore specimens were assigned to fixation groups based on DEXA values. [35] Unlike a previous report suggesting that female dogs had higher bone mineral density compared to males, [8] there was no significant difference in DEXA values between genders in our study. However, gender distribution was heavily weighted toward intact male dogs and results may be different if a larger number of dogs would be evaluated.

Previous studies have documented a high violation rate (up to 92\%) of vertebral canal, intervertebral foramen or vertebral foramen when using bicortical pins in the canine cervical vertebral column. $[6,8]$ We had $100 \%$ canal violation with the use of bicortical pins even though insertion landmarks and angles were followed. The incidence of canal violation by bicortical pins in clinical patients is unknown. Offending pins are likely not recognized as such because survey radiographs are inaccurate at predicting implant position in relation to the vertebral canal.[36] Postoperative CT is recommended to better assess pin placement in clinical patients.

Despite the goal of monocortical implant position of the screw/PMMA constructs, 7/72 screws $(9.7 \%)$ were found to be penetrating into the vertebral canal on visual inspection. 
Inadvertent bicortical drilling likely occurred before placement of those screws. The use of special drill stops or guides may have prevented accidental drilling through the transcortex but these were not used in our study. Clinically, the use of some form of drill stop is strongly recommended to not only avoid screw penetration but, more importantly, to prevent spinal cord injury by the drill bit. It is also feasible to consider that the use of self-tapping screws causes a higher incidence of screw penetration as $5 / 7$ penetrating screws were self-tapping. The highest incidence of screw penetration into the canal was of the screw in the middle of C4 vertebral body. This particular screw engaged the least amount of vertebral body bone of all the screws used in this construct. Based on this, one should be particularly careful when placing screws in the center of the vertebral body and an alternative location (i.e., closer to the cranial vertebral endplate) may be safer. Seven of 72 screws (9.7\%) caused a minor cortical lift without visible screw tip. It is suspected that this was because of the screw pushing against the transcortex with enough force to cause fracturing of the thin sheath of cortical bone making up the floor of the vertebral canal. It is unlikely that the inadvertent bicortical placement of some screws influenced the biomechanical data. In most specimens, it was 1 screw/construct that showed penetration and penetration was often minor, making purchase in the transcortex of questionable biomechanical advantage within our study.

Radiographic accuracy in predicting vertebral canal violation of bicortical implants is poor.[36] The accuracy of radiographic assessment of monocortical vertebral body screws has not been previously determined despite studies using survey radiographs as the determining modality to judge canal penetration.[9] Sensitivity and specificity for monocortical screw position on radiographs were high if screws were excluded that cause only cortical lift; however, since a minor cortical bone lift is of questionable clinical relevance, accuracy in detecting such violation seems of little importance and therefore radiographs appear to be a useful tool in detecting canal violation by monocortical screws.

A weakness of our study was that none of the constructs were fatigue tested. In a clinical setting long-term cyclic loading of implants is likely responsible for implant loosening and failure. We do not know how the monocortical screw construct would compare to the bicortical pin construct during such testing. Also, we did not investigate the effects of disk altering procedures at the treated VMU or the effects of stabilization on adjacent VMUs. It is assumed; however, that rigid fixation of $1 \mathrm{VMU}$ with constructs we used would have similar effects on adjacent VMUs as previously reported.[9, 34]

The ideal stiffness of implants for vertebral column stabilization is unknown. We used the stiffness of an established fixation method (bicortical pins/PMMA) to evaluate a monocortical screw construct. Whereas results indicate that all 3 constructs were significantly stiffer than the unaltered vertebral motion units, data were obtained in an in vitro model, which can never entirely represent biomechanical behavior in a living patient.

\section{ACKNOWLEDGMENT}

We thank Synthes for donation of screws. This study was funded by a Canine Intramural Grant of the Ohio State University College of Veterinary Medicine. 


\section{DISCLOSURE}

The authors report no financial or other conflicts of interest related to this report.

\section{REFERENCES}

1. Bergman RL, Levine JM, Coates JR, et al: Cervical spinal locking plate in combination with cortical ring allograft for a one level fusion in dogs with cervical spondylotic myelopathy. Vet Surg 2008; 37:530-536

2. Blass CE, Waldron DR, Van Ee RT: Cervical stabilization in three dogs using steinmann pins and methyl methacrylate. J Am Anim Hosp Assoc 1988; 24:61-68

3. Ellison GW, Seim HB III, Clemmons RM: Distracted cervical spinal fusion for management of caudal cervical spondylomyelopathy in large-breed dogs. J Am Vet Med Assoc 1988; 193:447-453

4. Voss K, Steffen F, Montavon PM: Use of the compact unilock system for ventral stabilization procedures of the cervical spine: a retrospective study. Vet Comp Orthop Traumatol 2006; 19:21-28

5. Bruecker KA, Seim HB, Blass CE: Caudal cervical spondylomyelopathy: decompression by linear traction and stabilization with steinmann pins and polymethyl methacrylate. J Am Anim Hosp Assoc 1989; 25:677-683

6. Koehler CL, Stover SM, LeCouteur RA, et al: Effect of a ventral slot procedure and of smooth or positive-profile threaded pins with polymethylmethacrylate fixation on intervertebral biomechanics at treated and adjacent canine cervical vertebral motion units. Am J Vet Res 2005; 66:678-687

7. Corlazzoli D: Bicortical implant insertion in caudal cervical spondylomyelopathy: a computed tomography simulation in affected Doberman Pinschers. Vet Surg 2008; 37:178-185

8. Hicks DG, Pitts MJ, Bagley RS, et al: In vitro biomechanical evaluations of screw-barpolymethylmethacrylate and pin-polymethylmethacrylate internal fixation implants used to stabilize the vertebral motion unit of the fourth and fifth cervical vertebrae in vertebral column specimens from dogs. Am J Vet Res 2009; 70:719-726

9. Agnello KA, Kapatkin AS, Garcia TC, et al: Intervertebral biomechanics of locking compression plate monocortical fixation of the canine cervical spine. Vet Surg 2010; 39:991-1000

10. Trotter EJ: Cervical spine locking plate fixation for treatment of cervical spondylotic myelopathy in large breed dogs. Vet Surg 2009; 38:705-718

11. Steffen F, Voss K, Morgan JP: Distraction-fusion for caudal cervical spondylomyelopathy using an intervertebral cage and locking plates in 14 dogs. Vet Surg 2011; 40:743-752

12. Bruecker KA, Seim HB III, Withrow SJ: Clinical evaluation of three surgical methods for treatment of caudal cervical spondylomyelopathy of dogs. Vet Surg 1989; 18:197-203

13. Shamir MH, Chai O, Loeb E: A method for intervertebral space distraction before stabilization combined with complete ventral slot for treatment of disc-associated wobbler syndrome in dogs. Vet Surg 2008; 37:186-192

14. Hueftle MG, Modic MT, Ross JS, et al: Lumbar spine: postoperative MR imaging with GdDTPA. Radiology 1988; 167:817-824

15. Wang JC, Sandhu HS, Yu WD, et al: MR parameters for imaging titanium spinal instrumentation. J Spinal Disord 1997; 10:27-32 
16. Cha JG, Jin $\mathrm{W}$, Lee $\mathrm{MH}$, et al: Reducing metallic artifacts in postoperative spinal imaging: usefulness of IDEAL contrast-enhanced T1- and T2-weighted MR imaging - phantom and clinical studies. Radiology 2011; 259:885-893

17. Rupp R, Ebraheim NA, Savolaine ER, et al: Magnetic resonance imaging evaluation of the spine with metal implants. General safety and superior imaging with titanium. Spine (Phila Pa 1976) 1993; 18:379-385

18. Knott PT, Mardjetko SM, Kim RH, et al: A comparison of magnetic and radiographic imaging artifact after using three types of metal rods: stainless steel, titanium, and vitallium. Spine J 2010; 10:789-794

19. Christensen FB, Dalstra M, Sejling F, et al: Titanium-alloy enhances bone-pedicle screw fixation: mechanical and histomorphometrical results of titanium-alloy versus stainless steel. Eur Spine J 2000; 9:97-103

20. Stambough JL, Genaidy AM, Huston RL, et al: Biomechanical assessment of titanium and stainless steel posterior spinal constructs: effects of absolute/relative loading and frequency on fatigue life and determination of failure modes. J Spinal Disord 1997; 10:473-481

21. Bruecker KA, Seim HB III: Principles of spinal fracture management. Semin Vet Med Surg (Small Anim) 1992; 7:71-84

22. Watine S, Cabassu JP, Catheland $S$, et al: Computed tomography study of implantation corridors in canine vertebrae. J Small Anim Pract 2006; 47:651-657

23. Swaim SF: Evaluation of four techniques of cervical spinal fixation in dogs. J Am Vet Med Assoc 1975; 166:1080-1086

24. Spivak JM, Chen D, Kummer FJ: The effect of locking fixation screws on the stability of anterior cervical plating. Spine (Phila Pa 1976) 1999; 24:334-338

25. United States EPA. Methyl methacrylate-interim acute exposure guideline levels. Office Pollut Prevent Toxics 2008; http://www.epa.gov/oppt/aegl/pubs/methyl_methacrylate_interim_oct_2008_v2001.pdf

26. Mjoberg B, Pettersson $H$, Rosenqvist $R$, et al: Bone cement, thermal injury and the radiolucent zone. Acta Orthop Scand 1984; 55:597-600

27. Meyer PR Jr, Lautenschlager EP, Moore BK: On the setting properties of acrylic bone cement. J Bone Joint Surg Am 1973; 55:149-156

28. Webb JC, Spencer RF: The role of polymethylmethacrylate bone cement in modern orthopaedic surgery. J Bone Joint Surg Br 2007; 89:851-857

29. Smith GK: Orthopaedic biomaterials. in Newton CD, Nunamaker DM (eds): Textbook of small animal orthopaedics. Philadelphia, J.B. Lippincott Co., 1985, pp 231-241

30. Poitout DG: Biomaterials used in orthopedics. in Poitout DG (ed): Biomechanics and biomaterials in orthopedics. London, UK, Springer, 2004, pp 15-21

31. Johnson AL, Houlton JEF, Vannini R: Implants and materials in fracture fixation. in $A O$ principles of fracture management in the dog and cat. Davos Platz, Switzerland, AO Publishing, Stuttgart, Germany, Thieme, 2005, pp 477-487

32. Leclet $\mathrm{H}$ : Artifacts in magnetic resonance imaging of the spine after surgery with or without implant. Eur Spine J 1994; 3:240-245

33. Ebraheim NA, Savolaine ER, Stitgen SH, et al: Magnetic resonance imaging after pedicular screw fixation of the spine. Clin Orthop Relat Res 1992; 279:133-137 
34. Adamo PF, Kobayashi H, Markel M, et al: In vitro biomechanical comparison of cervical disk arthroplasty, ventral slot procedure, and smooth pins with polymethylmethacrylate fixation at treated and adjacent canine cervical motion units. Vet Surg 2007; 36:729-741

35. Halvorson TL, Kelley LA, Thomas KA, et al: Effects of bone mineral density on pedicle screw fixation. Spine (Phila Pa 1976) 1994; 19:2415-2420

36. Hettlich BF, Fosgate GT, Levine JM, et al: Accuracy of conventional radiography and computed tomography in predicting implant position in relation to the vertebral canal in dogs. Vet Surg 2010; 39:680-687 